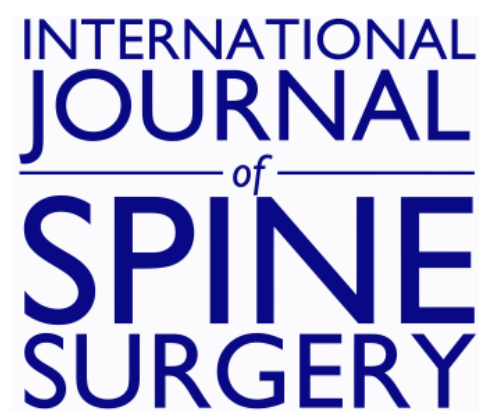

\title{
Patient Selection in Cervical Disc Arthroplasty
}

Pierce Nunley, Kelly Frank and Marcus Stone

Int J Spine Surg 2020, 14 (s2) S29-S35

doi: https://doi.org/10.14444/7088

http://ijssurgery.com/content/14/s2/S29

This information is current as of April 26, 2023.

Email Alerts Receive free email-alerts when new articles cite this article. Sign up at: http://ijssurgery.com/alerts 


\title{
Patient Selection in Cervical Disc Arthroplasty
}

\author{
PIERCE NUNLEY, MD, KELLY FRANK, MS, MARCUS STONE, PHD \\ Spine Institute of Louisiana, Shreveport, Louisiana
}

\begin{abstract}
Background: Patient selection for cervical disc arthroplasty (CDA) in the United States remains a topic of debate among surgeons. Many surgeons base US patient selection for CDA implantation on the Food and Drug Administration (FDA) indications/contraindications. While off-label use does occur, the frequency and extent of offlabel use in the US remains largely unknown. Outside the United States, patient selection is notably less stringent; however such data also remain largely unpublished or presented/published with a low level of evidence. Here, we will review the current approved US on-label patient selection criteria for CDA and discuss the rationale and supporting evidence to expand these criteria in the United States.

Methods: A PubMed literature search was completed using the keywords "cervical disc arthroplasty" and "cervical disc replacement." The articles were evaluated by the authors for patient selection criteria.

Conclusions: The current published data do not conclusively prove that the patients excluded from CDA by strict adherence to FDA indications would benefit from CDA surgery over anterior cervical discectomy and fusion. As surgeons, it is a difficult decision regarding when to expand indications to include off-label use of CDA. In our practice, generally CDA patient selection agrees with the FDA indications and contraindications, as there is a lack of level 1 evidence to confirm effectiveness of CDA outside of the current FDA indications. We will likely need more wellconstructed studies to include prospective and controlled trials that specifically evaluate the "off-label" applications before US surgeons are convinced to expand indications and insurance companies agree to reimburse.
\end{abstract}

Special Issue-Cervical Spine

Keywords: cervical spine surgery, off-label spine surgery, surgical indications, TDR, total disc replacement

\section{INTRODUCTION}

Food and Drug Administration (FDA)-approved investigational device exemption (IDE) studies have been conducted to gain approval for each cervical disc arthroplasty (CDA) device. The FDA used the strict inclusion and exclusion criteria derived from these IDE studies to develop appropriate indications and contraindications for CDA. While patient selection for most CDA implantations in the United States are based on these FDA criteria (on-label use), there are patients who receive CDA where the indications/contraindications are not strictly adhered to (off-label use).

The frequency and extent of off-label use for CDA in the United States is unknown, and patient selection remains a topic of debate among surgeons. Currently, any systematic off-label use in the United States remains largely unpublished.

Outside the United States (OUS), CDA patient selection is known to be notably less stringent; however, such data also remain largely unpublished or presented/published with a low level of evidence.
AOSpine attempted to understand the worldwide use of CDA by conducting an international survey of its members (6179) in 2016. Only 383 questionnaires were completed for analysis, representing 67 countries. Results indicated that $84.3 \%$ of surgeons continue to use anterior cervical discectomy and fusion (ACDF) for cervical disc herniation, 47.8\% occasionally use CDA, and only $7.3 \%$ use CDA as the standard treatment. The most important reasons for not using CDA were cost and low evidence for benefit, while less concerning were complications, legal concerns, and insurance. ${ }^{1}$

In 2016, Park et $\mathrm{al}^{2}$ reviewed 21 failed CDA patients who underwent revision surgery within 2 years. CDA failure was defined as the persistence of recurrence of clinical symptoms due to residual or new pathologies at the index level. The authors concluded that the primary cause for failure was patient selection $(81 \%)$. The next most common cause requiring revision was failure of technique, citing insufficient decompression and malposition as the major technical errors. This led to secondary 
Table 1. Common indications and contraindications (patient selection criteria) of the approved US IDE CDA devices.

Indications

- Single or two contiguous levels between C3 and C7 for conditions:

- Intractable radiculopathy (with or without neck pain)

Or myelopathy

- And at least one of the following:

- Herniated nucleus pulposus

Spondylosis (defined by osteophytes)

- Visible loss of disc height compared to adjacent levels

- Skeletally mature patient

- Failure of 6 wk of conservative care

Contraindications

- Axial neck pain only

- Prior cervical spine surgery, including prior surgery at the index level

- More than 2 diseased levels requiring surgery

- Segmental instability

- Severe spondylosis

- Disc height $<3 \mathrm{~mm}$

- Severe facet joint degeneration

- Significant kyphotic deformity

- Compromised vertebral body at index level due to prior trauma or significant abnormality or disease

- Osteoporosis

Abbreviations: CDA, cervical disc arthroplasty; IDE, investigational device exemption.

issues, such as eccentricity, subsidence, altered kinematics, and recurrence of pathologies.

The authors recommended careful patient selection, including patients with soft disc herniation or mild spondylosis, and provided an algorithmic approach to revision CDA. ${ }^{2,3}$ This study highlights clearly that indications and contraindications used in most parts of the United States differ from those used OUS. This creates a "gray zone" regarding the pathologies that benefit from ongoing motion preservation. Can all grades of disc degeneration be treated with CDA? Or is CDA restricted to early degenerative changes with neural compressive disease? Which pathology lies within the gray zone that can be successfully be treated with CDA? The authors will review the current approved US onlabel patient selection criteria for CDA and discuss the rationale and supporting evidence to expand these criteria.

\section{Current US Patient Selection Criteria for CDA}

Although the modern use of CDA began in 2000 OUS, adoption of CDA in the United States was marked by the FDA approval of Prestige ST in 2007. ${ }^{4}$ The Prodisc-C and Bryan disc approvals followed shortly thereafter in 2007 and 2009, respectively. ${ }^{5,6}$ Between 2012 and 2016, 4 additional discs received FDA approval: Secure-C, PCM, Mobi-C, and Prestige LP. Both the Mobi-C and
Prestige LP discs received approval for 1- and 2level indications, the only current 2-level approvals in the United States. ${ }^{7-12}$

The FDA approval process for CDA requires an IDE with strict inclusion and exclusion criteria. On approval, the FDA publishes indications, contraindications, warnings, and precautions for each device. The FDA uses the clinical trial inclusion/ exclusion criteria to determine the appropriate indications/contraindications. The indications/contraindications, while similar, are not the same for each CDA device. ${ }^{13-21}$ Table 1 illustrates the most common (although not inclusive) indications/contraindications across CDA devices. It is generally accepted that patients meeting these criteria are candidates for CDA.

In 2004, $\mathrm{McAfee}^{22}$ reported that "the indications for anterior cervical disc replacement (CDA) are the same as for anterior cervical decompression: radiculopathy or myelopathy caused by either one or two levels of anterior cervical compression." Generally, the contraindications reported here are like those in Table 1, implying that there has been little change in surgeon perception of CDA patient selection since 2004.

In 2012, Ding and Shaffrey ${ }^{23}$ reported some case illustrations on CDA patient selection that resulted in poor outcomes. Poor patient selection consisted of patients with disc space collapse greater than $50 \%$ (resulting in overdistraction), axial neck pain (specifically when motion was preserved where facet arthropathy existed), older age (pathologies were more prominent), and preoperative loss of segmental motion (CDA is for motion preservation, not motion restoration). The authors concluded that CDA should be considered for patients with singlelevel disease, anterior or disc-related pathology, preserved segmental motion, preserved disc space height, no significant facet arthropathy, and normal sagittal alignment. Fusion should be considered in cases of multilevel disease, combined anterior and posterior pathology, loss of segmental motion, collapsed disc space, segmental ankylosis, osteoporosis, significant kyphosis, segmental instability, tumor, trauma, infection, and previous decompressive laminectomies. ${ }^{23}$

Auerbach et $\mathrm{al}^{24}$ used the US on-label criteria for Prestige, Prodisc-C, PCM, and Bryan to analyze patients requiring cervical spine surgery. There were 167 patients identified as requiring cervical surgery, and 72 were qualified, meeting all indications and no 
Table 2. Inclusion and exclusion criteria from Brazil PCM study. ${ }^{25}$

\section{Inclusion criteria}

- 18-80 y old

- Discogenic radiculopathy of the cervical spine, with radiculopathy symptoms in 1 or both arms; pain, paresthesias, or paralysis in a specific nerve root distribution

- Involvement at 1, 2, 3, or 4 disc levels from C3 to T1

- Radiographically determined discogenic radiculopathy to include at least one of the following:

- Cervical spondylosis or cervical spondylitic myelopathy

- Disc herniation on CT or MRI

- Pseudarthrosis or failed prior attempted ACDF

Failed cervical cages or arthroplasty

- 6 wk conservative care

Exclusion criteria

- Prior disc space infection or osteomyelitis in the cervical spine

- Previous trauma to the $\mathrm{C} 3-\mathrm{C} 7$ levels resulting in compression or bursting

- Axial neck pain in the absence of other symptoms of radiculopathy

- Osteoporosis, osteopenia, or other metabolic bone disease of the spine

- Active malignancy or other spinal tumor

- Acute cervical trauma or instability: no anterior subluxation $>3.5$ mm on flexion-extension radiographs

- Circulatory, cardiac, or pulmonary problems that could cause excessive surgical risk

- Known or suspected metal allergy

- Severe myelopathy to the extent that the patient is wheelchair bound

- Mid-sagittal stenosis of $<8 \mathrm{~mm}$, as measured by CT or MRI

- Autoimmune disorders (eg, lupus, rheumatoid arthritis)

- Psychosocial disorder (indicated by Waddell score $>3$ )

- Morbid obesity, defined as body mass index $>40$ or more than 100 lb over ideal body weight

Abbreviations: ACDF, anterior cervical discectomy and fusion; CT, computed tomography; MRI, magnetic resonance imaging.

contraindications. Analysis of contraindications of the 95 nonqualified patients indicated that 47 required surgery at more than 2 levels, 18 had prior surgery at the index level, and 7 required surgery adjacent to a prior fusion. The data presented are compelling with less than $50 \%$ of patients qualifying for a CDA using the current on-label criteria. ${ }^{24}$ OUS has routinely used less rigid selection criteria since the approval of CDA, but the publications are limited. Currently, one of the most important questions is whether the rigidity in patient selection is helping or harming our patients and whether it is time for a shift in the US adoption of expanded indications for CDA.

\section{OUS Experience}

The OUS regulatory environment does not include published patient selection criteria, as is standard with US FDA approvals. Although limited, there are OUS publications on CDA that give insight into the OUS patient selection criteria. A Brazilian study of $\mathrm{PCM}^{25}$ and a French study of
Table 3. Inclusion and exclusion criteria from the France Mobi-C study. ${ }^{26}$

Inclusion criteria

- Degenerative disc disease at one or more levels between C3 and T1 confirmed with x-rays, CT, or MRI

- Radiculopathy and/or myelopathy

- Appropriate conservative medical treatment

Exclusion criteria

- Age $>65$ years old

- Noncompliance with study protocol

- Osteoporosis

- Metabolic bone disease

- Congenital or posttraumatic deformity

- Infection

- Neoplasia

- Instability of the intersomatic space

- Narrow canal (<12 mm)

Abbreviations: CT, computed tomography; MRI, magnetic resonance imaging.

Mobi- $\mathrm{C}^{26}$ enrolled patients with inclusion and exclusion criteria far less strict than current US patient selection criteria (Tables 2 and 3). We will review in detail these commonly expanded patient selection criteria and supporting literature.

\section{Commonly Expanded Patient Selection Criteria}

Some indications/contraindications are not heavily debated, such as osteoporosis, severe facet degeneration, prior cervical spine surgery, and significant loss of disc height, while others elicit varied opinions from surgeon users. We selected the more debated indications/contraindications to evaluate the state of the literature and surgeon perception.

\section{More Than 2 Levels (Including Noncontiguous Levels)}

To date, no US IDE study has included patients with cervical degenerative disc disease at more than 2 contiguous levels. ${ }^{4-12}$ There are multiple publications, mostly OUS, that have reported CDA use at 3 or more levels, often including noncontiguous levels.

In Brazil, CDA (PCM) data through 3 years was favorable for patients treated up to 4 levels. As expected, the number of patients treated at 3 and 4 levels was low: 12 patients with 3 levels and 4 with 4 levels. While the study inclusion criteria were less restrictive than US studies, there were similarities in exclusions (Table 2). ${ }^{25}$ Studies from Asia are similar, with Zhang et $\mathrm{al}^{27}$ reporting good clinical outcomes and no serious complications through 4 years for patients receiving Bryan at 2 noncontiguous levels. Zhao et $\mathrm{a}^{28}$ reported on the Bryan CDA at up to 3 levels. The 10-year results included favorable clinical outcomes but high rates of heterotopic ossification. 
The Mobi-C CDA was used in a similar study in France, with 4 patients treated at 3 levels and 1 patient treated at 4 levels, with favorable results through a 2-year follow-up. Again, similarities to the US studies were noted in the study exclusion criteria (Table 3). ${ }^{26}$ The critique remains that these studies were low-level evidence and prospective, nonrandomized, and low enrollment. Based on the paucity of evidence in the literature, it appears that surgeon perception of the use of CDA at more than 2 levels remains mostly unfavorable in the United States. However, Gornet et $\mathrm{al}^{29}$ provide a report in this focus issue with data from 139 patients suggesting that 3- and 4-level CDA may be performed safely and effectively in appropriately selected patients. ${ }^{29}$

\section{Hybrid}

CDA with fusion hybrids fall into 2 categories: (1) CDA implanted simultaneous (same surgery) adjacent to a fusion or (2) CDA adjacent to a prior fusion. There is a paucity of data on simultaneous hybrid implantations, although many surgeons support the concept. The most common rationale is treating 2 contiguous levels where 1 level meets the criteria for CDA and the other does not. Proponent surgeons claim to also use it for biomechanical considerations in certain patients. Mo et $\mathrm{al}^{30}$ reported on biomechanical considerations of multiple CDA devices selected in hybrid surgery for bilevel cervical degenerative disc disease using finite element analysis. The authors concluded that Prodisc-C, Mobi-C, and Discover performed similarly with respect to spinal motion, adjacent intradiscal pressures, and driving moments, while Bryan and PCM were more comparable biomechanically. This leads the authors to suggest hybrid construct with the use of Bryan and PCM for patients with potential risk of facet joint degeneration and with Prodisc-C, Mobi-C, and Discover for patients with risk of vertebral osteoporosis.

There is a limited literature reporting on the implantion of CDA adjacent to a prior fusion. The Brazilian study conducted with PCM also included implantation adjacent to a prior fusion (hybrid). PCM was used in 12 1-level and 9 multilevel cases adjacent to a previous fusion with favorable results. $^{25}$ The FDA trial for PCM also allowed patients with prior cervical fusions, including adjacent to the CDA. The success data for these patients were presented separately but do not distinguish patients with CDA adjacent to fusion versus noncontiguous. ${ }^{7}$ While not directly contraindicated by the FDA, prior cervical fusion at any cervical level was published as an FDA precaution. The FDA establishes a precaution when the safety and effectiveness of a device have not been established for patients with this condition. ${ }^{16}$

Prior cervical fusions were also not excluded from the French Mobi-C study, with 21 1-level patients and 5 multilevel patients with prior fusions. Eighteen 1-level patients and 3 multilevel patients received a CDA adjacent to the fusion. ${ }^{26}$ However, the hybrid results are unable to be specifically interpreted from either the Mobi-C or the PCM study, as the data were presented as single level versus multilevel only.

The data on CDA simultaneous with fusion are only biomechanical in nature, while the data on CDA adjacent to a prior fusion are not presented with enough detail for interpretation. US surgeon perception remains largely unfavorable for both CDA hybrid constructs.

\section{Revision of Failed Fusion}

In addition to hybrid constructs, the PCM study from Brazil and the Mobi-C study from France included CDA implants as a revision to a failed fusion. The PCM study included 11 1-level and 9 multilevel CDAs as a revision to a failed fusion, and the Mobi-C study included 2 patients. The results from both studies were favorable; however, the data for these specific patients were not reported separately. ${ }^{25,26} \mathrm{CDA}$ used as a revision to a failed fusion remains controversial in the United States, with little to no published data on this subset of patients.

\section{Kyphosis}

While the US studies specifically exclude severely kyphotic patients from enrollment (IDE studies), the OUS studies did not exclude these patients (Tables 2 and 3). However, this population was not analyzed separately in the OUS studies, so understanding the severity and prevalence of kyphosis preoperatively and the outcomes in that population is not possible with the data presented.

However, there was a post hoc analysis of the US Mobi-C data that analyzed patients with radiographically identified postoperative kyphosis, and there were no differences in clinical outcomes. ${ }^{31}$ Yoon et $\mathrm{al}^{32}$ found in a study in Korea that patients 
treated with Bryan adjacent to a fusion experienced postoperative kyphotic change. These differences also did not impact clinical outcomes.

Staudt et $\mathrm{al}^{33}$ recently analyzed the different CDA designs for single-piece versus multipiece, fixed versus mobile center of rotation, constraint type, materials, fixation, compression, and sagittal balance. While their article does not directly address CDA patient selection, it begins to explore the idea of patient-specific implants. Specifically, the authors discuss the incorporation of implant lordosis for CDA devices. ${ }^{33-37}$ Currently, only early data on lordotic implants have been collected for the Discover disc and the Synergy disc with varying results.

Regardless of the lack of literature on CDA and kyphosis, many OUS surgeons believe that the best sagittal balance is achieved by allowing the cervical spine to move, effectively finding its own balance. ${ }^{25}$ Limited literature does report that postoperative kyphotic changes in CDA patients do not impact outcomes, so perhaps preoperative exclusion is unnecessary. However, in general, US surgeons remain unconvinced and eliminate patients with kyphosis as candidates for CDA.

\section{Other Considerations}

While the indications and contraindications discussed previously are the most commonly reported and debated, there are many recent publications that address other factors to consider in CDA patient selection. Multiple publications indicate that preoperative pain, demographics, comorbidities, litigation, workers' compensation, and psychosocial factors affect outcomes in lumbar and cervical spine surgery. ${ }^{38-43}$ Gornet et $\mathrm{al}^{44}$ reported in 2013 that higher baseline neck pain and SF-36 MCS were significantly associated with successful outcomes in CDA patients, although they did not find a relationship to workers' compensation status in this cohort.

The number and variations of CDA devices available make the idea of selecting a CDA that is most appropriate for the patient's condition a reality. However, to achieve this, the strengths and weaknesses of each CDA device need to be understood.

\section{Cost and Reimbursement}

The debate to expand CDA use in the United States cannot ignore device costs and the current reimbursement landscape. Since its approval in the United States by the FDA, often the decision to implant a CDA is highly influenced by insurance approval, sometimes requiring on-label use. However, in addition to the literature overwhelmingly confirming the safety and efficacy of CDA, cost analyses indicate that it is a clear cost advantage in

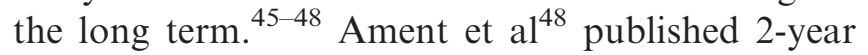
data that indicated that an incremental costeffectiveness ratio of CDA to ACDF was $\$ 24,594$ per quality-adjusted life year, well below the established threshold of $\$ 50,000$ per quality-adjusted life year. That said, the off-label or expanded uses discussed here remain even more difficult to obtain insurance approval for.

\section{CONCLUSIONS}

Although more than $50 \%$ of patients diagnosed with cervical degenerative disc disease are being excluded $^{24}$ by strict adherence to on-label use of CDA, current published data do not conclusively prove that those would benefit from CDA surgery over ACDF. For surgeons, it is a difficult decision on when to expand indications to include off-label use of CDA. In our practice, generally CDA patient selection agrees with the FDA indications and contraindications. We find that 3-level disease is commonly diagnosed in clinical practice, but there is lack of level 1 evidence to confirm effectiveness of 3level CDA over ACDF. The CDA hybrid solution is also lacking conclusive evidence of its effectiveness. We will likely need more well-constructed studies to include prospective and controlled trials that specifically evaluate the off-label applications before US surgeons are convinced to expand indications and insurance companies agree to reimburse.

\section{REFERENCES}

1. Chin-See-Chong TC, Gadjradj PS, Boelen RJ, Harhangi BS. Current practice of cervical disc arthroplasty: a survey among 383 AOSpine International members. Neurosurg Focus. 2017;42(2):E8. doi: 10.3171/2016.11.FOCUS16338

2. Park JB, Chang H, Yeom JS, Suk KS, Lee DH, Lee JC. Revision surgeries following artificial disc replacement of cervical spine. Acta Orthop Traumatol Turc. 2016;50:610-618.

3. Skovrlj B, Lee DH, Caridi JM, Cho SK. Reoperations following cervical disc replacement. Asian Spine J. 2015;9(3):471-482.

4. US Food and Drug Administration. Summary of Safety and Effectiveness Data (SSED): Prestige Cervical Disc. Washington, DC: US Food and Drug Administration; 2007.

5. US Food and Drug Administration. Summary of Safety and Effectiveness Data (SSED): ProDisc-C Total Disc Replace- 
ment. Washington, DC: US Food and Drug Administration; 2007.

6. US Food and Drug Administration. Summary of Safety and Effectiveness: Bryan Cervical Disc. Washington, DC: US Food and Drug Administration; 2009.

7. US Food and Drug Administration. Summary of Safety and Effectiveness Data (SSED): PCM Cervical Disc. Washington, DC: US Food and Drug Administration; 2012.

8. US Food and Drug Administration. Summary of Safety and Effectiveness: Secure-C Cervical Artificial Disc. Washington, DC: US Food and Drug Administration; 2012.

9. US Food and Drug Administration. Summary of Safety and Effectiveness Data (SSED): Mobi-C Cervical Disc Prosthesis (One-Level). Washington, DC: US Food and Drug Administration; 2013.

10. US Food and Drug Administration. Summary of Safety and Effectiveness Data (SSED): Mobi-C Cervical Disc Prosthesis (Two-Level). Washington, DC: US Food and Drug Administration; 2013.

11. US Food and Drug Administration. Summary of Safety and Effectiveness Data (SSED): Prestige LP Cervical Disc (One-Level). Washington, DC: US Food and Drug Administration; 2014.

12. US Food and Drug Administration. Summary of Safety and Effectiveness Data (SSED): Prestige LP Cervical Disc (Two-Level). Washington, DC: US Food and Drug Administration; 2016.

13. US Food and Drug Administration. Product Labeling for Prestige Cervical Disc. Washington, DC: US Food and Drug Administration; 2007.

14. US Food and Drug Administration. Product Labeling for Prodisc-C Cervical Disc. Washington, DC: US Food and Drug Administration; 2007.

15. US Food and Drug Administration. Product Labeling for Bryan Cervical Disc. Washington, DC: US Food and Drug Administration; 2009.

16. US Food and Drug Administration. Product Labeling for PCM Cervical Disc. Washington, DC: US Food and Drug Administration; 2012.

17. US Food and Drug Administration. Product Labeling for Secure-C Cervical Artificial Disc. Washington, DC: US Food and Drug Administration; 2012.

18. US Food and Drug Administration. Product Labeling for Mobi-C Cervical Disc. Washington, DC: US Food and Drug Administration; 2013.

19. US Food and Drug Administration. Product Labeling for Mobi-C Cervical Disc (Two-Level). Washington, DC: US Food and Drug Administration; 2013.

20. US Food and Drug Administration. Product Labeling for Prestige LP Cervical Disc (One-Level). Washington, DC: US Food and Drug Administration; 2014.

21. US Food and Drug Administration. Product Labeling for Prestige LP Cervical Disc (Two-Level). Washington, DC: US Food and Drug Administration; 2016.

22. McAfee PC. The indications for lumbar and cervical disc replacement. Spine J. 2004;4:177S-181S.

23. Ding D, Shaffrey ME. Cervical disk arthroplasty: patient selection. Clin Neurosurg. 2012;59:91-97.

24. Auerbach JD, Jones KJ, Fras CI, Balderston JR, Rushton SA, Chin KR. The prevalence of indications and contraindications to cervical total disc replacement. Spine J. 2008;8:711-716.

25. Pimenta L, McAfee PC, Cappuccino A, Cunningham BW, Diaz R, Coutinho E. Superiority of multilevel cervical arthroplasty outcomes versus single-level outcomes: 229 consecutive PCM prostheses. Spine (Phila Pa 1976). 2007;32:13371344.

26. Huppert J, Beaurain J, Steib JP, et al. Comparison between single- and multi-level patients: clinical and radiological outcomes 2 years after cervical disc replacement. Eur Spine J. 2011;20:1417-1426.

27. Zhang Z, Gu B, Zhu W, Wang Q, Zhang W. Clinical and radiographic results of Bryan cervical total disc replacement: 4year outcomes in a prospective study. Arch Orthop Trauma Surg. 2013;133:1061-1066.

28. Zhao Y, Zhang Y, Sun Y, Pan S, Zhou F, Liu Z. Application of cervical arthroplasty with Bryan cervical disc: 10-year follow-up results in China. Spine (Phila Pa 1976). 2016;41:111-115.

29. Gornet MF, Schranck FW, Sorensen KM, Copay AG. Multi-level cervical disc arthroplasty: long-term outcomes at 3 and 4 levels. IJSS. 2020;14(suppl 2):[this issue].

30. Mo Z, Li Q, Jia Z, Yang J, Wong DW, Fan Y. Biomechanical consideration of prosthesis selection in hybrid surgery for bi-level cervical disc degenerative diseases. Eur Spine J. 2017;26:1181-1190.

31. Hisey M, Davis R, Hoffman G, et al. Sagittal Alignment of One-Level TDR and ACDF Patients: An Analysis of Patient Outcomes From a Randomized, Prospective, Clinical Trial. Miami, FL: International Society for the Advancement of Spine Surgery; 2014.

32. Yoon DH, Yi S, Shin HC, Kim KN, Kim SH. Clinical and radiological results following cervical arthroplasty. Acta Neurochir (Wien). 2006;148:943-950.

33. Staudt MD, Das K, Duggal N. Does design matter? Cervical disc replacements under review. Neurosurg Rev. 2018;41:399-407.

34. Pickett GE, Mitsis DK, Sekhon LH, Sears WR, Duggal N. Effects of a cervical disc prosthesis on segmental and cervical spine alignment. Neurosurg Focus. 2004;17(3). doi: 10.3171/foc. 2004.17.3.5

35. Pickett GE, Rouleau JP, Duggal N. Kinematic analysis of the cervical spine following implantation of an artificial cervical disc. Spine (Phila Pa 1976). 2005;30:1949-1954.

36. Sears WR, Duggal N, Sekhon LH, Williamson OD. Segmental malalignment with the Bryan cervical disc prosthesis-contributing factors. J Spinal Disord Tech. 2007;20:111117.

37. Sears WR, Sekhon LH, Duggal N, Williamson OD. Segmental malalignment with the Bryan cervical disc prosthesis - does it occur? J Spinal Disord Tech. 2007;20:1-6.

38. Anderson PA, Subach BR, Riew KD. Predictors of outcome after anterior cervical discectomy and fusion: a multivariate analysis. Spine (Phila Pa 1976). 2009;34:161-166.

39. Aalto TJ, Malmivaara A, Kovacs F, et al. Preoperative predictors for postoperative clinical outcome in lumbar spinal stenosis: systematic review. Spine (Phila Pa 1976). 2006;31:E648-E663.

40. Cauthen JC, Kinard RE, Vogler JB, et al. Outcome analysis of noninstrumented anterior cervical discectomy and 
interbody fusion in 348 patients. Spine (Phila Pa 1976). 1998;23:188-192.

41. Skolasky RL, Riley LH, Albert TJ. Psychometric properties of the Cervical Spine Outcomes Questionnaire and its relationship to standard assessment tools used in spine research. Spine J. 2007;7:174-179.

42. BenDebba M, Heller J, Ducker TB, Eisinger JM. Cervical spine outcomes questionnaire: its development and psychometric properties. Spine (Phila Pa 1976). 2002;27:21162123; discussion 2124.

43. Garvey TA, Transfeldt EE, Malcolm JR, Kos P. Outcome of anterior cervical discectomy and fusion as perceived by patients treated for dominant axial-mechanical cervical spine pain. Spine (Phila Pa 1976). 2002;27:1887-1895; discussion 1895.

44. Gornet M, Schranck F, Taylor B, Kopjar B. Predictors of success after cervical disc arthroplasty. Spine J. 2013;13(9). doi: $10.1016 /$ j.spinee.2013.07.255

45. McAnany SJ, Overley S, Baird EO, et al. The 5-year costeffectiveness of anterior cervical discectomy and fusion and cervical disc replacement: a Markov analysis. Spine (Phila Pa 1976). 2014;39:1924-1933.

46. Radcliff K, Zigler J. Costs of cervical disc replacement versus anterior cervical discectomy and fusion for treatment of single-level cervical disc disease: an analysis of the Blue Health
Intelligence database for acute and long-term costs and complications. Spine (Phila Pa 1976). 2015;40:521-529.

47. Radcliff K, Lerner J, Yang C, Bernard T, Zigler JE. Seven-year cost-effectiveness of ProDisc-C total disc replacement: results from investigational device exemption and postapproval studies. J Neurosurg Spine. 2016;24:760-768.

48. Ament JD, Yang Z, Nunley P, Stone MB, Kim KD. Cost-effectiveness of cervical total disc replacement vs fusion for the treatment of 2-level symptomatic degenerative disc disease. JAMA Surg. 2014;149:1231-1239.

Corresponding Author: Marcus Stone, $\mathrm{PhD}$, Spine Institute of Louisiana, Shreveport, LA, 71101. Phone: (318) 629-5585; Email: mstone@ louisianaspine.org.

Published 30 September 2020

This manuscript is generously published free of charge by ISASS, the International Society for the Advancement of Spine Surgery. Copyright (c) 2020 ISASS. To see more or order reprints or permissions, see http://ijssurgery.com. 